\title{
Sustainable polymer composites: functionality and applications
}

\author{
Fengwei Xie ${ }^{*}$ (0)
}

Due to the alarming environmental issues and our heavy reliance on finite resources, sustainability has been globally recognised as one of the most important goals of our research and technology development. Given this, sustainable materials, especially those based on renewably and responsibly sourced or synthesised polymers, have attracted extensive interest. One major class of these sustainable polymers are biopolymers that are directly extracted from nature (including polysaccharides such as cellulose, chitin/chitosan, starch, and alginate and proteins such as collagen/gelatin, silk fibroin, keratin, and soy protein). Sustainable polymers also include polymers that are biologically or chemically synthesised from renewable sources of monomers, such as polyhydroxyalkanoates (PHAs) and polylactide (PLA). Besides, polymer chemistry development has reached the level where even a wider range of polymers, which are traditionally built with fossil fuels, can be synthesised partially or even fully from renewable feedstocks (biomass and carbon dioxide), such as polyglycolide (PGA), poly(butylene succinate) (PBS), polyolefins (polyethylene (PE) and polypropylene (PP)), poly(ethylene terephthalate) (PET), poly(ethylene furanoate) (PEF), polyamides (PAs), polyurethanes (PUs), and polycarbonates (PCs) [1-3]. Materials based on some sustainable polymers are biodegradable under composting conditions (e.g. PLA and PBS), in the natural environment (e.g. PHA and almost all biopolymers), or in the living body (e.g. chitosan, starch, PLA, PGA, and $\mathrm{PBS}$ ), while the other sustainable polymers are inherently recalcitrant to degradation (e.g. polyolefins, PET, PEF, and PAs), for which suitable end-of-life strategies

*Correspondence: d.xie.2@warwick.ac.uk; fwhsieh@gmail.com International Institute for Nanocomposites Manufacturing (IINM), WMG, University of Warwick, Coventry CV4 7AL, UK (e.g. recycling) must be implemented for minimising their environmental impact. Based on their degradability, along with varied properties and functionality, sustainable polymers can be developed into materials tailored for certain applications. Furthermore, the smart design of sustainable polymer composites through materials chemistry and engineering can endow such materials with appealing functionality, making them have enormous potential for important applications such as in packaging, health, and environmental areas. This special collection focuses on how new and enhanced functions can be realised by sustainable polymer composites, which are formed by mainly polymer blending, inclusion of small amounts of nanofillers, or loading with active ingredients, coupled with chemical functionalisation or specialised materials fabrication procedures.

Packaging materials for food needs to be biodegradable or compostable as food-contaminated packages are hard to be recycled, and be able to extend the shelf-life of food to avoid food waste [4]. As an important biopolymer from biomass, starch can be converted into so-called "thermoplastic starch" and has been extensively studied for materials applications [5-7] and especially for food packaging $[8,9]$. In this special collection, Villar et al. (UNS, UBA, and UNLu, Argentina) [10] demonstrated the antimicrobial capacity of corn starch-based films containing copper $(\mathrm{Cu})$ particles (DOI: https://doi.org/ 10.1186/s42252-020-00009-7). Regardless of particle type (without or with silica coating) or concentration (0.25$0.75 \mathrm{wt} \%)$, copper effectively migrated to a simulant of aqueous foods, inhibiting the growth of Gram-positive and Gram-negative bacteria (S. aureus and E. coli). Thus, this type of starch-based composite films can be useful for food primary packaging mainly for meat fresh products. 
Biopolymers such as chitosan have been extensively used in biomedical areas due to their biodegradability, biocompatibility and inherent antimicrobial activity [11]. In this special collection, Rehman et al. (Lancaster University, UK) [12] described the use of a freeze-gelated chitosan/hydroxyapatite porous scaffold to load heparin for the promotion of angiogenesis (DOI: https://doi.org/10. 1186/s42252-020-00012-y). It was noted that low heparin concentrations exhibited a positive effect, with approximately $28 \mu \mathrm{g}$ per scaffold indicating a significant increment in blood vessels. Besides, the synthesized materials showed no cytotoxic effects. Such a material holds promising potential for clinical use as a pro-angiogenic bone regeneration material.

This special collection also contains our research at the University of Warwick, UK [13] into the structure and properties of chitosan and chitosan/carboxymethyl cellulose $(\mathrm{CMC})$ composites with 2D graphene oxide (GO) or reduced GO (rGO) combined with 1D sepiolite (SPT) or cellulose nanocrystals (CNCs) (DOI: https://doi.org/ 10.1186/s42252-021-00026-0). The results highlight that multiple factors (e.g. filler geometry, filler surface chemistry, and polymer-filler interactions) determine material structure and properties. Interestingly, a 3D interconnected microstructure composed of GO and SPT due to the strong interactions between these hydrophilic nanofillers was observed. For the chitosan/CMC matrix, GO andSPT interacted synergistically increasing the mechanical properties of the matrix. This work can be insightful for the future design of biopolymer composites with tailored properties.

Carbon-based adsorbents possess exceptional adsorption capability, thus highly useful for removing contaminants from the environment; and in this area, sustainable biomass-derived carbon materials are especially interesting [14-16]. In this special collection, Hakkarainen et al. (KTH, Sweden) [17] described free-standing threedimensional lignin-derived porous graphene oxide (GO)based nanocomposites with excellent adsorption capacity for heavy metal ions and cationic dyes (DOI: https://doi. org/10.1186/s42252-020-00008-8). Specifically, graphene oxide (GO) was obtained by hydrothermal carbonization and oxidation of lignosulfonate and contained abundant oxygen functionalities. Then, the self-assembly of this GO and gelatin following freeze-drying enabled the construction of porous composites. Functionalization of the porous composite with lignosulfonate could further enhance its adsorption performance $(66 \mathrm{mg} \mathrm{Cu}(\mathrm{II}) / \mathrm{g}$ and $38 \mathrm{mg}$ methylene blue/g).

Foams represent an important class of materials due to their low material usage, lightweight, and high surface area. However, the massive use of foamed products of petroleum-based polymers (e.g. polystyrene (PS), poly(vinyl chloride) (PVC), and PU) that are not readily degradable have caused environmental concerns. PLA has been considered as a substitute for petroleum-based polymers to make foams for various applications from packaging, cushioning, insulation, plastic utensils, to biomedical scaffolds [18]. In this special collection, Sinha Ray et al. (CSIR, South Africa) [19] described a foam with $>80 \%$ porosity prepared from PLA, poly[(butylene succinate)-co-adipate] (PBSA), and cellulose nanocrystal $(\mathrm{CNC})$ particles by the casting and particulate leaching (CPL) method using fructose as a porogen (DOI: https:// doi.org/10.1186/s42252-020-00011-z). PBSA reduced the brittleness and increased the thermal stability of PLA. CNC was shown to suppress PLA crystallisation, increase thermal stability, and improve the stiffness of the blend foam.

PLA and its nanocomposites have been widely investigated for biomedical applications considering its biodegradability, biocompatibility, and good thermochemical properties $[20,21]$. The crystalline structure and crystallinity of PLA significantly affect its mechanical properties $[22,23]$ and thus its application potential. PLA crystallisation can be induced by nucleation agents [24]. This special collection contains a study led by Di Luccio and Kornfield (Caltech, USA) [25] on the effect of tungsten disulphide $\left(\mathrm{WS}_{2}\right)$ nanotubes as a filler on the crystallisation of the PLA films at various stages of processing (unstretched, stretching, annealing) (DOI: https://doi. org/10.1186/s42252-021-00016-2). They found that $0.6 \mathrm{wt} \%$ of $\mathrm{WS}_{2}$ induced the same level of crystallinity in as stretched PLA-WS $\mathrm{W}_{2}$ as annealing in neat PLA for $10 \mathrm{~min}$. This work highlights the role of $\mathrm{WS}_{2}$ nanotubes

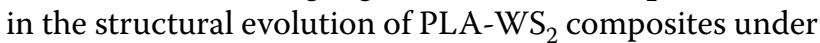
uniaxial deformation and provides an insight into the fine-tuning of PLA crystallinity.

I am thankful to all the contributors and reviewers to this special collection, and to the Editor-in-Chief for giving me the chance to edit this special collection. I believe that this special collection will serve as a valuable resource for research in sustainable polymer composites for packaging, biomedical, environmental, and other applications. Of course, the six papers contained in this special collection cannot represent the whole of the recent advances in this research area of topical interest, but it hopefully will stimulate further discussions and inspire more submissions to Functional Composite Materials in this area. Indeed, there are many challenges set before the sustainable polymer community, associated with e.g. batch-to-batch variability, cost-effectiveness, processibility, and material properties and functionality. Besides, there is still huge room to explore the science and engineering of sustainable polymers for developing advanced and functional materials in various forms (e.g. 
film, membrane, hydrogel, aerogel, and sophisticated constructs) for applications such as biomedical treatment, energy generation and storage, sensing, bioelectronics, insulation, and water treatment.

\section{Author's contributions}

The author wrote and approved the final manuscript.

\section{Declarations}

\section{Competing interests}

The author declares that there are no competing interests.

Published online: 21 October 2021

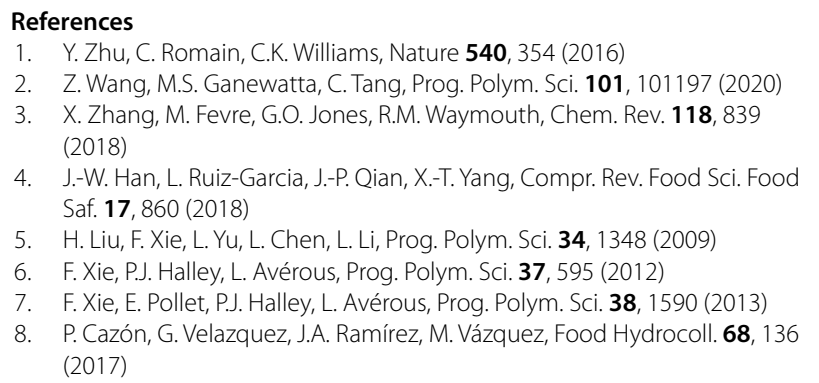

9. A.R.V. Ferreira, V.D. Alves, I.M. Coelhoso, Membranes 6, 22 (2016)

10. O.V. López, M.E. Villanueva, G.J. Copello, M.A. Villar, Funct. Composite Materials 1, 6 (2020)

11. M. Dash, F. Chiellini, R.M. Ottenbrite, E. Chiellini, Prog. Polym. Sci. 36, 981 (2011)

12. G.V. Nájera-Romero, M. Yar, I.U. Rehman, Funct. Composite Materials 1, 9 (2020)

13. P. Chen, F. Xie, F. Tang, T. McNally, Funct. Composite Materials 2, 14 (2021)

14. W. Ao et al., Renew. Sustain. Energy Rev. 92, 958 (2018)

15. M.M. Hassan, C.M. Carr, Chemosphere 265, 129087 (2021)

16. M. Danish, T. Ahmad, Renew. Sustain. Energy Rev. 87, 1 (2018)

17. J. Yao, K. Odelius, M. Hakkarainen, Funct. Composite Materials 1, 5 (2020)

18. M. Nofar, C.B. Park, Prog. Polym. Sci. 39, 1721 (2014)

19. M.P. Motloung, S. Zungu, V. Ojijo, J. Bandyopadhyay, S.S. Ray, Funct. Composite Materials 1,8 (2020)

20. M.S. Singhvi, S.S. Zinjarde, D.V. Gokhale, J. Appl. Microbiol. 127, 1612 (2019)

21. J.-M. Raquez, Y. Habibi, M. Murariu, P. Dubois, Prog. Polym. Sci. 38, 1504 (2013)

22. H. Simmons, P. Tiwary, J.E. Colwell, M. Kontopoulou, Polym. Degrad. Stab. 166,248 (2019)

23. L. Aliotta, P. Cinelli, M.B. Coltelli, M.C. Righetti, M. Gazzano, A. Lazzeri, Eur. Polym. J. 93, 822 (2017)

24. S. Saeidlou, M.A. Huneault, H. Li, C.B. Park, Prog. Polym. Sci. 37, 1657 (2012)

25. F. Loffredo, L. Tammaro, T. Di Luccio, C. Borriello, F. Villani, S. De Vito, K. Ramachandran, J.A. Kornfield, Funct. Composite Materials 2, 3 (2021)

\section{Publisher's Note}

Springer Nature remains neutral with regard to jurisdictional claims in published maps and institutional affiliations.

\section{Submit your manuscript to a SpringerOpen ${ }^{\odot}$ journal and benefit from:}

- Convenient online submission

- Rigorous peer review

- Open access: articles freely available online

- High visibility within the field

- Retaining the copyright to your article

Submit your next manuscript at $\boldsymbol{\nabla}$ springeropen.com 